\title{
PATRIMONIO EDFFICADO DE LA INMIGRACIÓN CHINA EN EL CENTRO HISTÓRICO DE LIMA ${ }^{(\star)}$
}

\author{
BUILT HERITAGE OF CHINESE IMMIGRATION IN HISTORICAL CENTER OF LIMA
}

\author{
ANGIE CHANG ESTRADA ${ }^{(* \star]}$ \\ (D) https://orcid.org/0000-0003-4837-2973 \\ achang0824@gmail.com \\ Universidad Nacional de Ingeniería (Perú) \\ Fecha de recepción: 1 noviembre de 2019 \\ Fecha de aprobación: 12 de agosto de 2020
}

\section{RESUMEN}

El presente articulo es una reflexión sobre el patrimonio edificado de la inmigración china en el Centro Histórico de Lima y de su consideración como tal. Para ello se ha investigado la relación de los sujetos patrimoniales con el patrimonio edificado de acuerdo con las nuevas posturas sobre patrimonio. La investigación presenta catorce casos agrupados como: (i) una zona monumental, (ii) un ambiente urbano monumental, (iii) diez edificios patrimoniales y (iv) dos hitos urbanos y los cruza con dos miradas: interna y externa para verificar la existencia del patrimonio edificado de la inmigración china en el Centro Histórico de Lima.

\section{PALABRAS CLAVE}

Patrimonio edificado; Centro Histórico de Lima; Inmigración china

\begin{abstract}
The article reflects on the consideration of the built heritage of the Chinese immigration in historical center of Lima. For this, the relationship between heritage subjects and built heritage has been studied taking as reference the new positions on heritage. The investigation presents fourteen cases, grouped as: (i)a monumental zone, (ii) a monumental urban space, (iii) ten heritage buildings, and (iv) two landmarks and crosses them with two outlooks: internal and external to verify the existence of the built heritage of Chinese immigration in the Historical Center of Lima.
\end{abstract}

\section{KEYWORDS}

Built heritage; Historical Center of Lima; Chinese immigration

\footnotetext{
(*) Este artículo se desarrolló a partir de la investigación titulada Patrimonio edificado de la Inmigración China en el Centro Histórico de Lima, realizada en el Taller de Investigación en Historia de la Arquitectura entre los años 2014 y 2018, bajo la responsabilidad del MSc. Arq. José Beingolea del Carpio, y luego del MSc. Arq. José Hayakawa Casas, en el marco académico de la Facultad de Arquitectura, Urbanismo y Artes de la Universidad Nacional de Ingeniería (UNI).

${ }^{(* *)}$ Bachiller en Arquitectura por la Universidad Nacional de Ingeniería. Participante del proyecto "La Ruta de los Patios culturales de Barrios de Altos". Primer puesto en el Concurso Centro de Investigación Facultad de Ingeniería Mecánica (FIM) de la Universidad Nacional de Ingeniería (2018), Mención Honrosa en el Concurso Plaza de las Artes Fundación Banco de la Nación con Balarq Consultores (2008). Actualmente se desempeña como Consultora externa en Sistemas integrados de gestión en el rubro de la construcción.
} 
devenir Vol. 7, Nº14, JULIO-DICIEMBRE 2020, PP. 1155-172 - ESTUDIOS ISSN 2312-7562 E-ISSN 2616-4949

UNIVERSIDAD NACIONAL DE INGEENIERÍA, LIMA

doi: https://doi.org/10.21754/devenir.v7i14.836

Allá por el año 2014, mientras caminaba por los alrededores de la Calle Capón en Barrios Altos, me di con la sorpresa de que la fachada de la emblemática Sociedad PunYui -que por aquellas épocas prestaba sus ambientes en el segundo nivel para que el Grupo León (del cual formaba parte) practicara la danza del León y Dragón- había sido cruelmente modificada con la tosca construcción de una falsa fachada simulando un muro cortina con dos tiendas en sus extremos con puertas metálicas enrollables. A partir de ese momento, empecé a cuestionar por qué le ocurría esto a tan bello edificio - una de las Sociedades Chinas mejor conservadas- que yo previamente había estudiado a través del libro de Isabel Lausent-Herrera y en el cual baso mi muestra para esta investigación.

A partir de esta búsqueda de respuestas encontré dos grupos: el primero conformado por académicos que buscaban proteger las diferentes Sociedades Chinas convirtiéndolas en Patrimonio Cultural de la Nación y, por el otro lado, los propietarios que estaban preocupados por la sobrevivencia del predio, por lo cual consideraron necesaria la expansión de espacio comerciales para garantizar su subsistencia. ¿Cuál de los dos grupos tenía razón? El académico que buscaba la inscripción como Patrimonio ante el Ministerio de Cultura, el cual iba a imponer unas reglas de intangibilidad que el propietario encontraría insostenible y aceleraría la destrucción del predio o el propietario que, sin mala intención, hizo un cambio sin asesoría técnica restándole valor a su propio patrimonio. Sobre estas consideraciones comencé a repensar sobre el estado actual y futuro de los edificios patrimoniales de la inmigración China en el Centro Histórico de Lima ¿Eran dichos edificios importantes para la comunidad y para el país? Finalmente, acabé por cuestionar su existencia.

\section{La visión actual de patrimonio}

Al día de hoy, la posición de las instituciones gubernamentales respecto al patrimonio está apoyada en la visión antigua del concepto, donde el bien -mueble o inmueblees intangible, lo que trae como consecuencia la precariedad del bien.

La antigua visión era un acercamiento académico que desestimaba la relación individuo-bien lo que generaba la pérdida del patrimonio intangible y evitaba que se formaran conexiones vitales para su supervivencia. Según Feliu, los conceptos relacionados a Patrimonio son dinámicos y se están ampliando debido a que el comportamiento social ante el bien cultural no ha sido lo suficientemente analizado. (Feliu, 2014, p.11)

Ballart rescata la intangibilidad del bien en el concepto de valor patrimonial y lo clasifica en las categorías de valor de uso, valor formal y valor simbólico:

[En el] valor de uso, evaluamos el bien tanto que sirve para satisfacer alguna necesidad concreta, sea individual o colectiva, o dar respuesta a algún reto o oportunidad [...] Al considerar un valor formal, evaluamos el bien por la atracción que despierta en los sentidos y en función del placer estético y la emoción que proporciona, pero también en función de otros atributos difíciles de conceptuar, tales como rareza, preciosidad, apariencia exótica o genio (¿es el artista o la obra lo que es genial?) [...] Al considerar un valor simbólico, entramos en un terreno crucial para el especialista en ciencias humanas y sociales, puesto que pretendemos evaluar el bien en tanto que nos pone en relación con su creador o sus usuarios en el pasado (sabemos que todos los signos, y entre ellos los símbolos, sirven para designar y relacionar, evocar o representar). (2000, p.20-21)

Para McCarthy, (citado por Villamón, 2017, p.131) el término edificio patrimonial no implica obligatoriamente su declaración oficial, por el contrario, este refleja los valores y significados heredados de una comunidad que transforman al edificio en un símbolo, parte de su identidad. Howard (citado por Villamón, 2017, p. 129) también hace referencia a este aspecto manejando dos escalas de patrimonio: 
formal e informal. El patrimonio formal es el reconocido por las instituciones y el informal es el reconocido por los actores que tienen una relación directa con el edificio.

Así, esta investigación se estructura sobre la visión actual de patrimonio donde los valores patrimoniales y la concepción de patrimonio -formal e informal- son determinantes para definir qué es y no es patrimonio.

\section{El patrimonio de la Inmigración china}

Se conoce como Inmigración china al Perú al desplazamiento masivo ocurrido entre 1849 - 1930. En este flujo migratorio chino se da la coincidencia de sucesos en ambas costas del Pacífico. En el lado chino, el imperio de los Qing presentaba dificultades económicas y colonialistas, a la vez que acontecían rebeliones internas, como la del Reino Celestial de Taiping. Por su parte, en el Perú, primero la declaratoria de libertad de vientres, lo que mermó la cantidad de esclavos en el país y, en segundo lugar, el boom del guano y el auge de la agricultura en las haciendas para los que faltaban mano de obra.

Los chinos que arribaban al Perú lo hacían bajo contrato que los obligaba a trabajar por cinco años, luego extendidos a ocho, y que incluso permitía la recontrata. (Rodríguez, 2001, p. 46) Al termino de sus contratos, los chinos abandonaban las haciendas y se trasladaban a las ciudades aledañas estableciéndose principalmente en zonas comerciales con el fin de buscar empleo. En el caso de la ciudad de Lima, se establecieron en los alrededores del Mercado de la Concepción -hoy Mercado Central- al coincidir con su construcción ordenada por Ramón Castilla.

Según la investigadora Isabelle Lausent-Herrera, los chinos se caracterizaban por ser una población de rápida organización gracias a los mecanismos migratorios propios de su lugar de origen, ya que, para poder movilizarse dentro de China, se formaban agrupaciones - denominadas huigan- de carácter clánico, lingüístico o por aldeas. En China, huigan permite al migrante tener un lugar de llegada en otros distritos, poder comunicarse en la misma lengua, contar con ayuda, etc. (2018)

Ya ubicados en el Centro de Lima y según sus costumbres los chinos se agruparon en distintos huigan, hoy denominados Sociedades Chinas'. Las agrupaciones más grandes alquilaban locales donde, bajo el sistema de apoyo mutuo propio de su cultura, daban soporte legal en relación a los contratos, vivienda temporal y-de suma importancia- la celebración de ritos y cultos.

Con el pasar de los años y la concentración de chinos en las quintas aledañas al Mercado Central -Callejón de Otayza, por ejemplo- se desarrollaron diferentes tipos arquitectónicos propios de la diversidad cultural: fondas, teatros chinos, lugares de juego que formarían el Barrio Chino de Lima.

De estos asentamientos originados por la migración, se abordan en este artículo catorce casos, presentados en cuatro grupos según su tipo: (i) una zona monumental: Barrio Chino; (ii) un ambiente urbano monumental: Calle Capón; (iii) diez edificios patrimoniales: Sociedad Tong Sing, Sociedad Cu Con Chau, Sociedad Pun Yui, Sociedad Lung Sin Sea, Sociedad Kai Ping, Sociedad Lung Kong Ko So, Sociedad Hok Shan, Sociedad Chung Shan Huy Cun, Sociedad Nam Joy y Sociedad Tong Huy Chong Koc; y (iv) dos hitos urbanos: Arco Chino y Fuente China (ver Figura 1).

1. Los huigan, como instituciones, se inscriben en Registros Públicos bajo la denominación de Sociedad, lo que lleva a que sean conocidas por los investigadores como Sociedades Chinas 


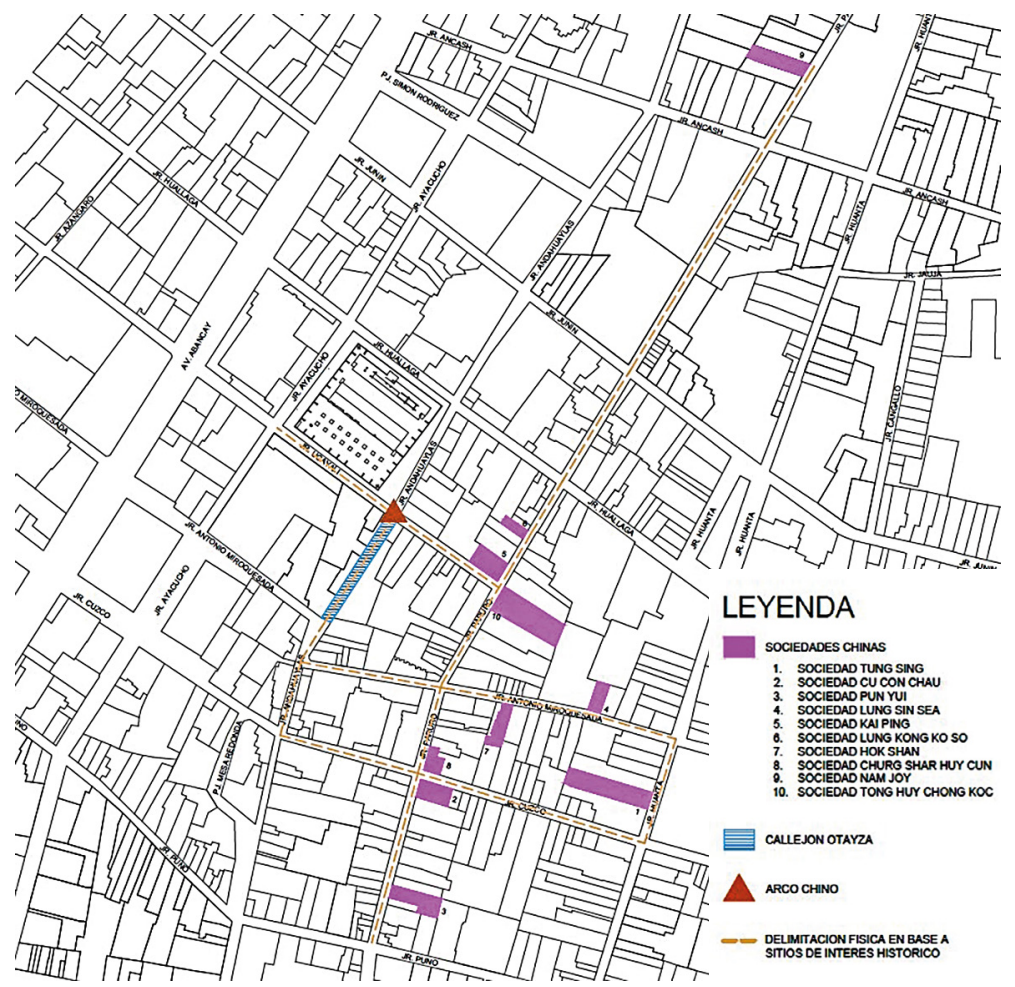

Figura 1. Ubicación de los catorce bienes estudiados. . Elaboración propia, 2018.

\section{Zona monumental}

Es la zona cuyo valor patrimonial, cultural, turístico y de recreación es reconocido y protegido; además de, concentrar viviendas y actividades urbanas complementarias. Este orden, otorgado por el Ministerio de Cultura, es aplicado aquí al Barrio Chino bajo la consideración actual de patrimonio citada párrafos arriba.

El Barrio Chino es un enclave étnico que representa la diáspora china en el mundo, el de Lima es uno de los barrios chinos más antiguos de América y el único barrio étnico del Perú. Según Lynch (2014) "las características físicas que determinan los barrios son continuidades temáticas que pueden consistir en una infinita variedad de partes integrantes como la textura, el espacio, la forma, los detalles, los símbolos," (p. ) entre otros; así, el Barrio Chino de Lima posee elementos decorativos de corte oriental (molduras, carteles e ideogramas) aplicados a las fachadas de corte occidental.

La población peruana reconoce con facilidad el Barrio Chino debido a su núcleo y a su hito característico -la Calle Capón y el Arco Chino respectivamente-; sin embargo, este barrio posee como parte de sus atractivos un mercado propio, varias Sociedades Chinas, diferentes comercios especializados y el oráculo de Tong Sing (ver Figura 2).

\section{Ambiente urbano monumental}

Es el espacio urbano que presenta una singularidad notable en su escala, fisonomía volumétrica y edificación de entorno con valor urbanístico de conjunto y que por ello debe ser conservado; así, al ambiente urbano monumental se le considera como "un espacio urbano que tiene calidad arquitectónica y urbanística. La calidad de ambiente Urbano monumental depende entonces tanto de cualidades arquitectónicas individuales, cuanto de efectos generales o de conjunto (armonía total o unidad)". (García Bryce citado por Hayakawa, 2012, p. 16) 


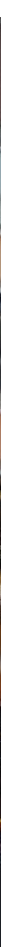

En tal sentido, se considera aquí a la Calle Capón -núcleo del Barrio Chino- como un ambiente urbano monumental que comprende las cuadras 6 y 7 del Jr. Ucayali en el centro histórico de Lima. Originalmente, estas dos cuadras comprendían una unidad y cuya división se originó al derrumbarse el Callejón de Otayza² provocando la ampliación del Jr. Andahuaylas. Al ubicarse el Arco Chino entre las cuadras 6 y 7 del Jr. Ucayali se reforzó la ruptura y, como consecuencia, el imaginario colectivo reconoce solo la cuadra 7 del Jr. Ucayali como la Calle Capón.

En el año 1999, para celebrar los 150 años de la presencia China en el Perú, se realizó la limpieza y remodelación de la Calle Capón -cuadra 7 del Jr. Ucayali-. Actualmente esta calle presenta baldosas conmemorativas de las que destacan doce grandes intervenciones con los animales del zodiaco chino, mobiliario en hierro con temática oriental y góndolas con techos inspirados en pagodas.

Alrededor de la Calle Capón están los principales comercios chinos, chifas y bancos, todos ellos con sus nombres traducidos al idioma chino; posee el ingreso al mercado chino y remata en la Sociedad Tong Huy Chong Koc conocida también como la Sociedad Central de Beneficencia China (ver Figura 3).

\section{Edificios patrimoniales}

Los edificios patrimoniales de la inmigración china como casuística son las denominadas Sociedades Chinas, agrupaciones de apoyo mutuo que poseen diferentes tipos edilicios, todos ellos occidentales con alguna adaptación oriental de diferente grado. Se les reconoce por tener principalmente un letrero rojo o un alto relieve en la parte
2. Según Rodríguez Pastor (2000) el Callejón de Otayza fue el nombre coloquial dado al espacio tugurizado por chinos en lo que alguna vez fue un jardín primero propiedad de Luis Otayza y posteriormente de una dueña de apellido Debernardi (p. 161)
Figura 2. El Barrio Chino de Lima. Archivo personal de la autora, fotografías tomadas por Yukio Ruiz Namisato, 2018

Figura 3. La Calle Capón. Archivo personal de la autora, fotografías tomadas por Yukio Ruiz Namisato, 2018.

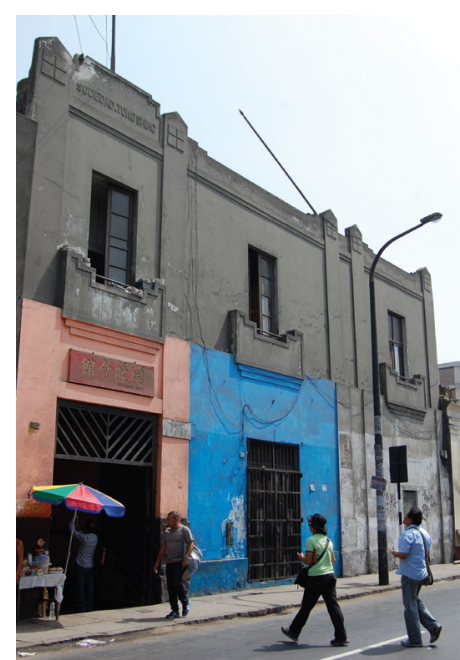

Figura 4. Fachada de la Sociedad Tong Shing. Archivo personal de la autora, fotografías tomadas por Yukio Ruiz Namisato, 2018 
de intervención del espacio con temática china, posee diferentes elementos ornamentales, módulos y colores característicos.

El uso actual de la Sociedad Tong Shing se divide por niveles: el primer nivel es residencial y el segundo nivel es religioso -templo oráculo donde se puede leer la fortuna a través del I Ching y alberga el anda de Guangong o San Acon que sale en procesión por año nuevo chino-y social -salón de reuniones- (ver Figuras 4 y 5 ).

\section{Sociedad Cu Con Chau}

La Sociedad Cu Con Chau es un huigan de lugar de procedencia:

"El nombre de la Ku y Kan o Guganzhou (Cu Con Chau) proviene de Gu (antiguo), de Jinggan (un pueblo del sur de la China) y de Zhou (que es una división administrativa correspondiente al departamento) [...] Basta saber que en la época Tang existía una provincia Ganzhou que desapareció y luego volvió a aparecer con el nombre de Gouganzhou. Incorporaba los cinco distritos (Taishan, Kaiping, Xinhui, Heshan, Enping)". (Lausent-Herrera, 2000, p.117).

El edificio actual de la Sociedad Cu Con Chau -inmueble de un nivel en esquina que posee un mezanine interior, adquirido en 1898 y remodelado en 1928 y $1972-$ se encuentra ubicado en el Jirón Paruro 1001 con Jr. Cuzco 800 en la zona de Barrios Altos en el distrito del Cercado de Lima y posee como área de lote $291.81 \mathrm{~m}^{2}$ aproximadamente. Actualmente es propiedad de la Asociación Civil Cu Con Chau.

Formalmente, la fachada del Jr. Puno presenta un cuerpo con un eje simétrico que remata en un entablamento corrido. Posee pilastras pareadas adosadas al paramento, falsos vanos con arco de medio punto, puerta de madera con arco de medio punto y reja en hierro forjado.
Figura 6. Fachada de la Sociedad Cu Con Chau. Archivo personal de la autora, fotografías tomadas por Yukio Ruiz Namisato, 2018. 
de las colecciones más antiguas de caligrafía china que tiene Perú- y el tercer nivel destinado a residencia (ver Figura 8).

\section{Sociedad Kai Ping}

La Sociedad Kai Ping es un huigan de lugar de procedencia que "reúne a los descendientes del pueblo de Kaiping (en dialecto cantonés Hoi Ping)". (Chuhue, 2012, p.440)

El edificio actual de la Sociedad Kai Ping -edificio contemporáneo de 4 niveles- se encuentra ubicado en el Jirón Ucayali 779, 781, 783, 787, 793 con intersección con Jirón Paruro, en la zona de Barrios Altos en el distrito del Cercado de Lima y posee como área de lote $664 \mathrm{~m}^{2}$ aproximadamente.

Formalmente su fachada presenta cuatro secciones dividas por sus respectivas losas, vanos

El uso actual de la Sociedad Kai Ping se divide por niveles: el primer nivel -posee una galería comercial y restaurantes con ingreso independiente de dos niveles-, el segundo nivel es comercial, el tercer para alquiler y cuarto nivel institucional (ver Figura 9).

\section{Sociedad Lung Kong Ko So}

La Sociedad Lung Kong Ko So es un huigan clánico que"fundada en octubre de 1919 por doce personas que pertenecían a cuatro clanes patronímicos. Los Liu, los Zhao, los Zhang y los Guan. Son los descendientes de los famosos héroes deificados de la Novela de [El Romance] los Tres Reinos". (Lausent-Herrera, 2000, p. 55).

El edificio actual de la Sociedad Lung Kong Ko So -edificio de cuatro niveles- se encuentra ubicado en el Jirón Paruro 756, 758, 760, en la zona de Barrios Altos, en el Cercado de Lima, y posee como área de lote $236 \mathrm{~m}^{2}$ aproximadamente.

Formalmente, la Sociedad Lung Kong Ko So está alineada al límite de propiedad y posee una fachada simétrica con una variante en el primer nivel donde se ubica el acceso a la escalera en el lado derecho. Se añaden además balcones de hierro en todos los pisos superiores.

El uso actual de la Sociedad Lung Kong Ko se divide por niveles: el primer nivel comercial -posee la tienda Hong Kong Market cuyo ingreso es independiente-, el segundo nivel es institucional y donde se brindan servicios de medicina alternativa, el tercer y cuarto nivel son viviendas probablemente usadas en la actualidad como almacenes (ver Figura 10).

\section{Sociedad Hok Shan}

La Sociedad Hok Shan es un huigan de lugar de procedencia:"en la actualidad los nativos y descendientes de Hok Shan (en mandarín He Shan) siguen realizando reuniones y actividades para sus asociados. Muchos de ellos proceden de Sha Ping, sede del gobierno regional". (Chuhue, 2012, p.440).

El inmueble original era una edificación con valor monumental cuya fachada fue modificada y posteriormente demolida. El edificio actual es una construcción posterior a 1996, tiene dos niveles y se encuentra ubicada en el Jirón Antonio Miroquesada 826, en la zona de Barrios Altos en el distrito del Cercado de Lima y posee como área de lote $542 \mathrm{~m}^{2}$ aproximadamente. Actualmente es propiedad de la Asociación de Auxilios Mutuos y Asistencia Social Ju San.

Formalmente su fachada presenta dos niveles y está alineada al límite de la propiedad, las puertas y rejas poseen carpintería metálica y las ventanas en carpintería de aluminio.

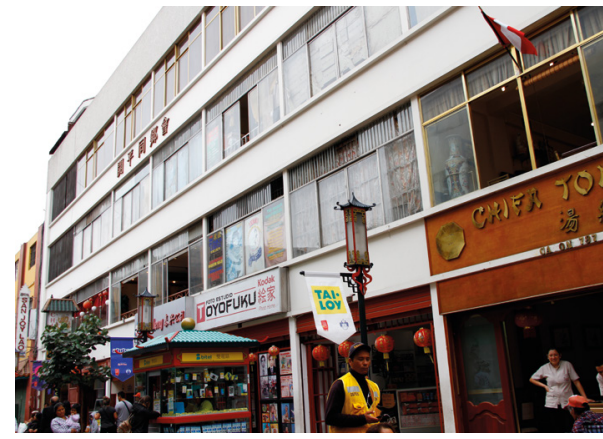

Figura 9. Fachada de la Sociedad Kai Ping. Archivo personal de la autora, fotografías tomadas por Yukio Ruiz Namisato, 2018.

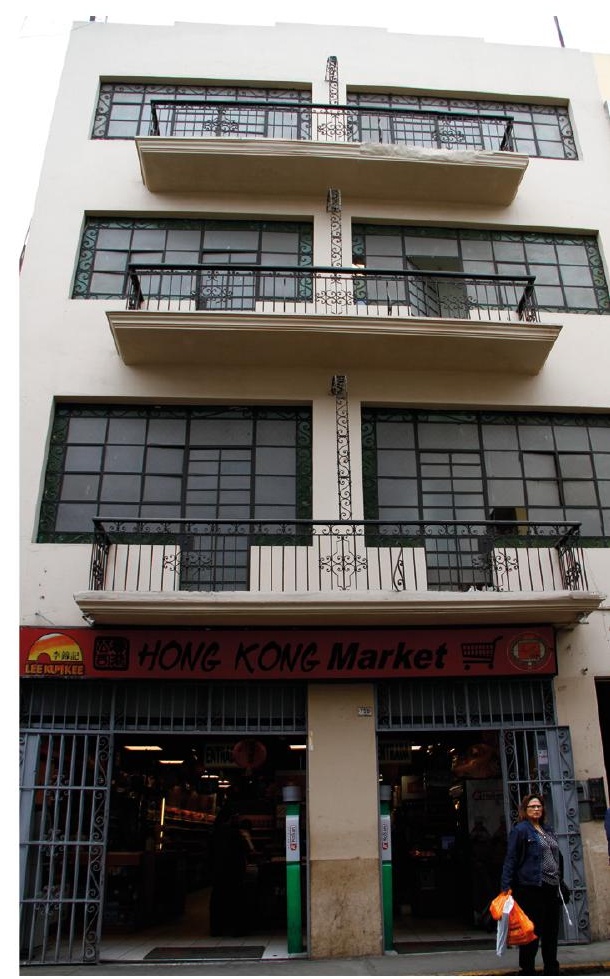

Figura 10. Fachada de la Sociedad Lung Kong Ko So. Archivo personal de la autora, fotografías tomadas por Yukio Ruiz Namisato, 2018 
devenir Vol. 7, Nº14, JULIO-DICIEMBRE 2020, PP. 1155-172 - EsTUDIOS ISSN 2312-7562 E-ISSN 2616-4949

UNIVERSIDAD NACIONAL DE INGGENIERÍA, LIMA

doi: https://doi.org/10.21754/devenir.v7i14.836

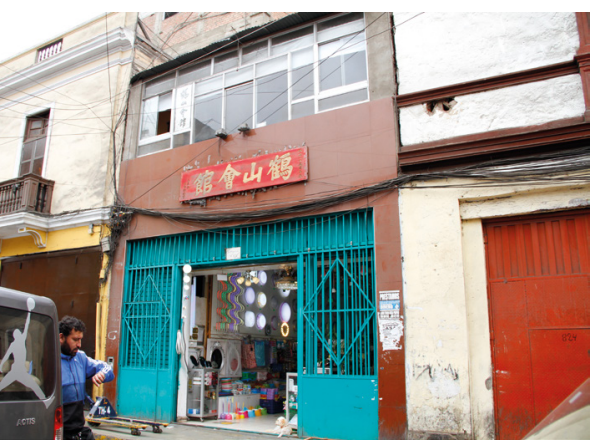

Figura 11. Fachada de la Sociedad Hok Shan. Archivo personal de la autora, fotografías tomadas por Yukio Ruiz Namisato, 2018.

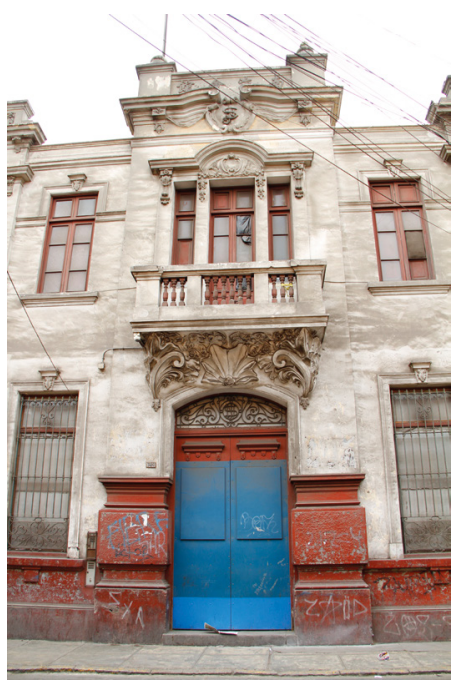

Figura 12. Fachada de la Sociedad Chung Shan Huy Cun. Archivo personal de la autora, fotografía tomada por Yukio Ruiz Namisato, 2018.

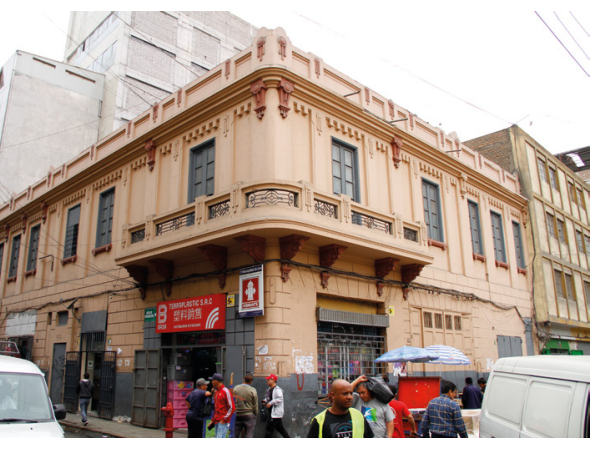

Figura 13. Fachada de la Sociedad Nam Joy. Archivo personal de la autora, fotografía tomada por Yukio Ruiz Namisato, 2018.
El uso actual de la Sociedad Hok Shan se divide por niveles: el primero comercial -con una tienda con ingreso independientes- y el segundo, institucional (ver Figura 11).

\section{Sociedad Chung Shan Huy Cun}

El edificio actual de la Sociedad Chung Shan Huy Cun -edificación en esquina de dos niveles- se encuentra ubicado en el Jirón Paruro 967, 975, 983, 991, 999 con intersección con el Jirón Cuzco, en la zona de Barrios Altos en el distrito del Cercado de Lima y posee como área de lote $348 \mathrm{~m}^{2}$ aproximadamente.

Formalmente la fachada de la Sociedad es ecléctica de influencias modernistas francesas.

El uso actual de la Sociedad Chung Shan Huy Cun se divide por niveles: el primer nivel comercial -posee varias tiendas con ingresos independientes-, el segundo nivel es institucional (ver Figura 12).

\section{Sociedad Nam Joy}

La Sociedad Nam Joy es un huigan de lugar de procedencia: "solía reunir a los inmigrantes de los pueblos vecinos a las riberas del delta del rio Las Perlas; Nan Joy, San Sei, Ya Yuen y Sung Tac, cada cual con su dialecto". (Chuhue, 2012, p.439).

El edificio actual es una edificación de dos niveles ubicado en el Jirón Paruro 340, 346, 352, en la zona de Barrios Altos en el distrito del Cercado de Lima y posee como área de lote $765.60 \mathrm{~m}^{2}$ aproximadamente. Actualmente es propiedad de Francisco Willy Celiz Aquino.

La Sociedad presenta una fachada ecléctica con elementos academicistas y modernistas, así como algunos detalles particulares, como las volutas sueltas a manera de frontón partido, propios de la tradición limeña.

El uso actual es residencial, en el pasado además de ser una Sociedad China fue sede del colegio de mujeres San Min (ver Figura 13).

\section{Sociedad Tong Huy Chong Koc}

La Sociedad Tong Huy Chong Koc también conocida como la Sociedad Central de Beneficencia China es un huigan de lugar de procedencia: "es la institución que agrupa a todos los residentes chinos en el país". (Chuhue, 2012, p.434).

El edificio actual de la Sociedad Central de Beneficencia China es una edificación de tres niveles remodelado y reinaugurado en 1959. Se encuentra ubicado en el Jirón Paruro 819 en el remate de la Calle Capón en la zona de Barrios Altos en el distrito del Cercado de Lima y posee como área de lote $1764 \mathrm{~m}^{2}$ aproximadamente. Actualmente es propiedad de la Sociedad Beneficencia China Dto Yaka Tong Ching.

Formalmente presenta una fachada moderna temprana, de influencia corbusiana con intento de ventana corrida horizontal,

El uso actual de la Sociedad Central de Beneficencia China se divide por niveles: el primer nivel es institucional, el cual alberga un salón multiusos, una biblioteca, oficinas y directorio. Los niveles superiores son de uso residencial. En el pasado además de ser una Sociedad China fue sede del colegio de varones Chung Wha (ver Figura 14).

\section{Hitos urbanos}

\section{Arco chino}

El Arco Chino es el ícono principal de la Calle Capón, fue un regalo de la colonia china por el sesquicentenario de la independencia, el diseño fue realizado por el arquitecto Kuoway Ruiz y posee 8 metros ancho por 13 metros de alto. 


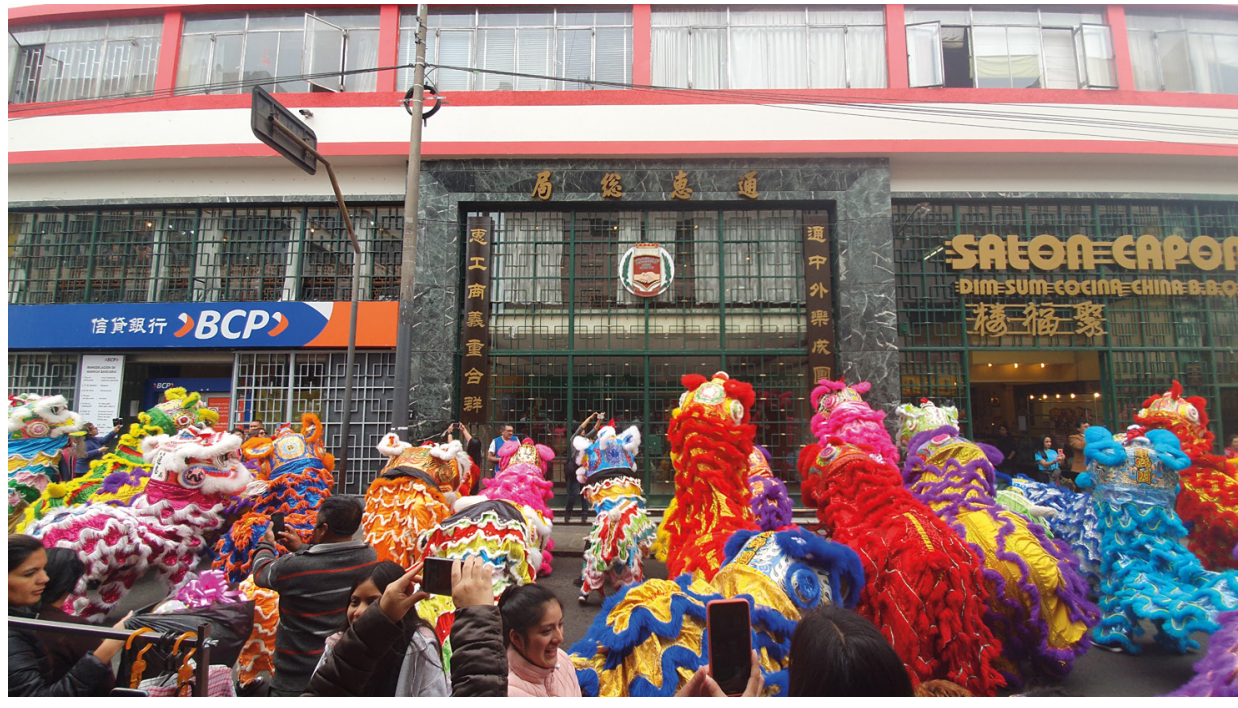

Figura 14. Fachada de la Sociedad Tong Huy Chong Koc Archivo fotográfico de la Sociedad de beneficencia china del Peru, 2018.

En la parte superior el arco tiene diversas inscripciones en idioma chino. En el lado que mira hacia la avenida Abancay se lee (en mayor dimensión y de derecha a izquierda) "El arco chino" [...]. En el lado que mira a la Calle Capón se lee una frase de la filosofía confucianista [...] "Bajo el mismo cielo, todos somos iguales". (Chuhue, 2016, p.63)

En la actualidad tiene a sus pies dos leones fu con rasgos africanos añadidos en el año 2017.

Sobre este hito, la arquitecta María Esther Ríos, menciona que "tiene impacto en la imagen de esta parte de la ciudad, inserto en un trazado histórico, con un valor más bien simbólico por ser el ingreso al Barrio Chino". (María Esther Ríos, comunicación personal, 8 de noviembre de 2018) (ver Figura 15)

\section{La Fuente china}

Emplazada en el Parque de la Exposición, la fuente China es un obsequio de la colonia china al Perú con motivo de las celebraciones del primer Centenario de la Independencia Nacional, es conocida también como la fuente de las Tres Razas al presentar en su cumbre una representación de las razas negra, amarilla y blanca. Diseñada por Gaetano Moretti y los escultores Giuseppe Graziosi y Valmore Gemignani, su factura es en mármol y bronce (ver Figura 16).

\section{Sujetos patrimoniales}

Los sujetos patrimoniales son diferentes grupos humanos que tienen relación directa con el objeto patrimonial a los cuales le otorgan valores en grado a su proximidad, uso, sentido de identidad y grado de reconocimiento. Los sujetos susceptibles al patrimonio edificado de la inmigración China en el centro histórico de Lima tienen dos miradas: la mirada interna que representa a la comunidad que deviene de esta inmigración (la comunidad china y la comunidad de los descendientes) y la mirada externa que representa a la comunidad peruana.

\section{Mirada interna - Comunidad china}

Los sujetos pertenecientes a este grupo proceden de las migraciones posteriores al establecimiento de las relaciones diplomáticas de la República Popular China con el

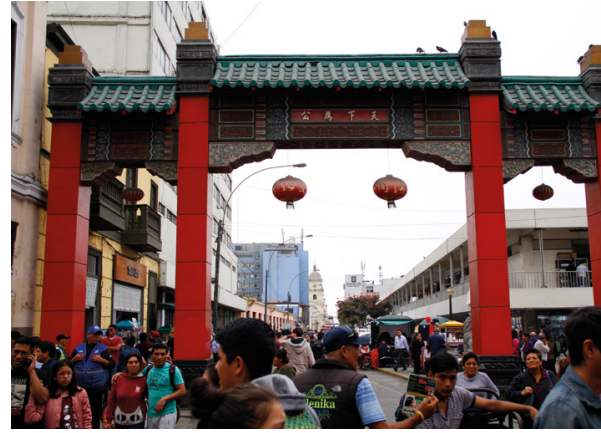

Figura 15. Arco chino. Archivo personal de la autora Archivo personal de la autora, fotografía tomada por Yukio Ruiz Namisato, 2018.

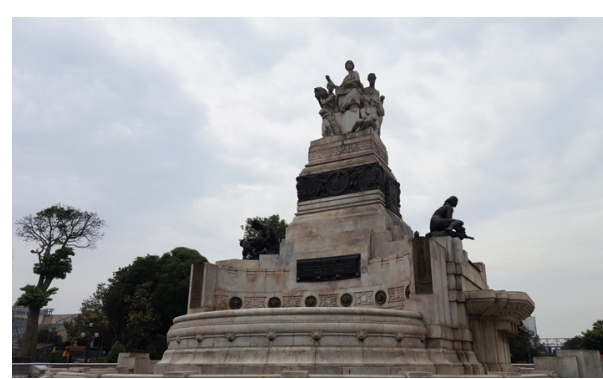

Figura 15. Fuente china Archivo personal de Oscar Huapaya, 2016. 
devenir Vol. 7, Nº14, JULIO-DICIEMBRE 2020, PP. 1155-172 - EsTUDIOS ISSN 2312-7562 E-ISSN 2616-4949

UNIVERSIDAD NACIONAL DE INGEEIERÍA, LIMA

doi: https://doi.org/10.21754/devenir.v7i14.836

\begin{tabular}{|c|c|c|}
\hline & Mirada interna & Mirada externa \\
\hline \multirow[t]{2}{*}{ Residentes } & Rubén Wong Robles & \\
\hline & Aldis Bernaola Siu & \\
\hline \multirow[t]{3}{*}{ Académicos } & Marco Loo Zambrano & Augusto Ortiz de Zevallos \\
\hline & Jesus Tang Tan & José Beingolea del Carpio \\
\hline & Miguel Situ Chang & Humberto Rodríguez Pastor \\
\hline \multirow[t]{2}{*}{ Interés Cultural } & Rodrigo Pinto de Sotta Campos & María Esther Ríos \\
\hline & Xuan Gan & \\
\hline
\end{tabular}

Perú, se caracterizan por ser pragmáticos, empresarios y cuyo dominio del idioma español es limitado.

- $\quad$ Mirada interna - Comunidad de los descendientes

Los sujetos pertenecientes a este grupo tienen al menos un ancestro chino cuyo origen está en la inmigración histórica (1849 - 1930), mantienen algunas tradiciones sino ninguna-, tienen una percepción nostálgica del país de sus ancestros y en algunos casos se agrupan buscando una identidad común.

- $\quad$ Mirada externa

Son todos aquellos que no presentan un vínculo con la migración china (ver Tabla 1).

\section{Valoracion patrimonial : discernimiento de la relacion objeto-sujeto}

La presente investigación es de tipo deductiva, se investigó desde lo general a lo específico presentando tres escalas: zona monumental, ambiente urbano monumental y monumento. Finalmente, con relación al método es cualitativa porque se enfoca en los valores patrimoniales, que se forman desde las diferentes relaciones objetos-sujetos.

\section{Herramientas metodológicas}

Divididas en dos etapas, la primera realizada en el año 2018 presenta (i) encuestas dirigidas a la mirada externa y público no especializado, se tomaron en puntos estratégicos del Centro Histórico de Lima incluido en Barrio Chino; (ii) tres tipos de entrevistas desarrolladas para el público con interés cultural, los académicos y los residentes; y (iii) documentación y registro: registro fotográfico realizado el año 2018, búsqueda en archivos y realización de fichas.

La segunda etapa realizada en el año 2020 presenta (i) el desarrollo de la matriz o rúbrica para cruzar la casuística contra los valores patrimoniales -tomados de Ballart en su extensión por Marsia Montañez-; y (ii) documentación y búsqueda en registros (ver Tabla 2).

\section{Análisis de resultados}

Según el análisis realizado en el 2018 y como se demuestra en la tabla Nº la casuística urbana -zona monumental, ambiente urbano monumental e hitos urbanospresenta valores patrimoniales más uniformes en comparación a la relacionada a 
Tabla 2. Rúbrica de valores de patrimoniales

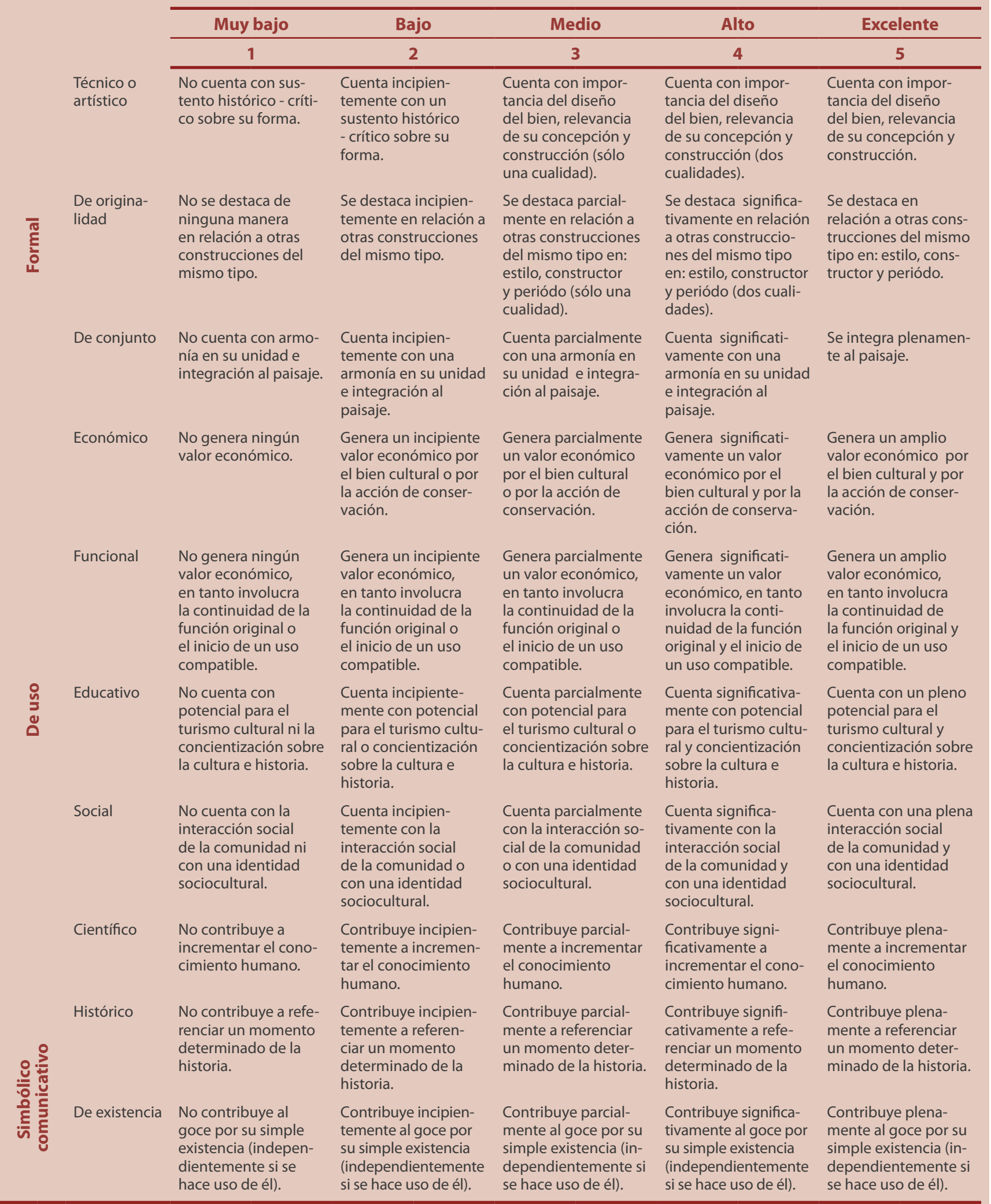

Elaboración propia en base a la tabla de valores patrimoniales de Marsia Montañez tomando como referencia los valores patrimoniales de Ballart y la guía del arquitecto Hayakawa, 2018. 
devenir Vol. 7, Nº14, JULIO-DICIEMBRE 2020, PP. 1155-172 - EsTUDIOS ISSN 2312-7562 E-ISSN 2616-4949

UNIVERSIDAD NACIONAL DE INGENIERÍA, LIMA

doi: https://doi.org/10.21754/devenir.v7i14.836

Tabla 2. Rúbrica de valores de patrimoniales

\begin{tabular}{|c|c|c|c|c|}
\hline Muy bajo & Bajo & Medio & Alto & Excelente \\
\hline 1 & 2 & 3 & 4 & 5 \\
\hline $\begin{array}{l}\text { No cuenta con valor } \\
\text { para las futuras gene- } \\
\text { raciones. }\end{array}$ & $\begin{array}{l}\text { Cuenta incipiente- } \\
\text { mente con valor para } \\
\text { las futuras genera- } \\
\text { ciones. }\end{array}$ & $\begin{array}{l}\text { Cuenta parcialmente } \\
\text { con valor para las } \\
\text { futuras generaciones. }\end{array}$ & $\begin{array}{l}\text { Cuenta significativa- } \\
\text { mente con valor para } \\
\text { las futuras genera- } \\
\text { ciones. }\end{array}$ & $\begin{array}{l}\text { Cuenta plenamente } \\
\text { con valor para las } \\
\text { futuras generaciones. }\end{array}$ \\
\hline $\begin{array}{l}\text { No cuenta con } \\
\text { reconocimiento } \\
\text { relacionado a los } \\
\text { lazos emocionales de } \\
\text { la sociedad. }\end{array}$ & $\begin{array}{l}\text { Cuenta incipiente- } \\
\text { mente con reconoci- } \\
\text { miento relacionado a } \\
\text { los lazos emocionales } \\
\text { de la sociedad. }\end{array}$ & $\begin{array}{l}\text { Cuenta parcialmente } \\
\text { con reconocimiento } \\
\text { relacionado a los } \\
\text { lazos emocionales de } \\
\text { la sociedad. }\end{array}$ & $\begin{array}{l}\text { Cuenta significativa- } \\
\text { mente con reconoci- } \\
\text { miento relacionado a } \\
\text { los lazos emocionales } \\
\text { de la sociedad. }\end{array}$ & $\begin{array}{l}\text { Cuenta plenamente } \\
\text { con reconocimiento } \\
\text { relacionado a los } \\
\text { lazos emocionales de } \\
\text { la sociedad. }\end{array}$ \\
\hline $\begin{array}{l}\text { No cuenta con una } \\
\text { imagen que diera } \\
\text { lugar a la evolución } \\
\text { histórica ni cambios } \\
\text { significativos del } \\
\text { bien. }\end{array}$ & $\begin{array}{l}\text { Cuenta incipiente- } \\
\text { mente con una ima- } \\
\text { gen que diera lugar a } \\
\text { la evolución histórica } \\
\text { o cambios significati- } \\
\text { vos del bien. }\end{array}$ & $\begin{array}{l}\text { Cuenta parcialmente } \\
\text { con una imagen } \\
\text { que diera lugar a la } \\
\text { evolución histórica o } \\
\text { cambios significati- } \\
\text { vos del bien. }\end{array}$ & $\begin{array}{l}\text { Cuenta significativa- } \\
\text { mente con una ima- } \\
\text { gen que diera lugar a } \\
\text { la evolución histórica } \\
\text { y cambios significati- } \\
\text { vos del bien. }\end{array}$ & $\begin{array}{l}\text { Cuenta plenamente } \\
\text { con una imagen } \\
\text { que diera lugar a la } \\
\text { evolución histórica y } \\
\text { cambios significati- } \\
\text { vos del bien. }\end{array}$ \\
\hline
\end{tabular}

Elaboración propia en base a la tabla de valores patrimoniales de Marsia Montañez tomando como referencia los valores patrimoniales de Ballart y la guía del arquitecto Hayakawa, 2018.

los monumentos -Sociedades Chinas-; debido a ello, el análisis se dividirá en dos secciones: Sobre las Sociedades y Sobre la zona monumental, el ambiente urbano monumental y los hitos urbanos.

Si observamos el análisis de los resultados del gráfico de araña, se observa que los tres valores patrimoniales -formal, uso y simbólico-significativo- se sobreponen, destacando ligeramente el valor de uso sobre los demás valores patrimoniales debido a la ubicación comercial de la muestra. Llama la atención que los monumentos presenten los índices más bajos en cuanto a reconocimiento de los valores patrimoniales; en contra posición, la casuística urbana tiene los índices más altos (ver Figura 17).

De la Tabla 3 se observa que seis casos de la muestra:-el Barrio Chino, la Calle Capón, Sociedad Central de Beneficencia China, Sociedad Pun Yui, Arco Chino y la Fuente Chinacuentan con al menos un valor; cuatro casos de la muestra -Sociedad Tong Shing, Sociedad Cu Con Chau, Sociedad Lung Sin Sea y Sociedad Chung Shang Huy Cun- tienen valor potencial; tres casos de la muestra -Sociedad Kai Ping, Sociedad Lung Kong Ko So y Sociedad Hok Shan- tienen escaso valor potencial; y un caso de la muestra-Sociedad Hok Shan- debido a sus últimas modificaciones ha perdido su valor.

\section{- Sobre las Sociedades Chinas}

\section{Valor formal - técnico o artístico}

Después de analizar las técnicas de investigación se concluye que la población peruana tiene una percepción negativa sobre la estética de las Sociedades, debido a que las fachadas muestran el deterioro de las mismas, el entorno descuidado a su vez contribuye al convencimiento de ello. Los académicos y los que presentan un interés cultural en el tema perciben un valor estético, tomando como referencia la Sociedad Pun Yui, la Sociedad Cu Con Chao y la Sociedad Tong Shing, además resaltan que esta última tiene un gran potencial.

Valor formal - de originalidad 


\section{Valores patrimoniales de los casos estudiados}

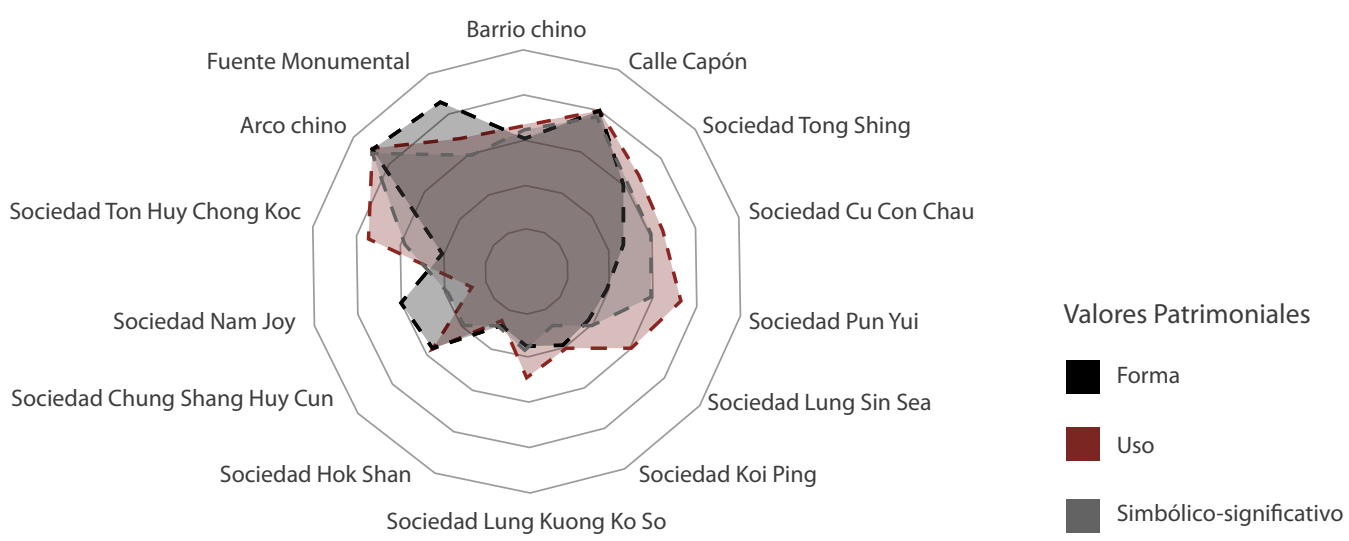

Figura 18. Valores patrimoniales de los casos estudiados. Elaboración propia, 2018.

Tabla 3. Casos de estudio versus valores patrimoniales - Resultados

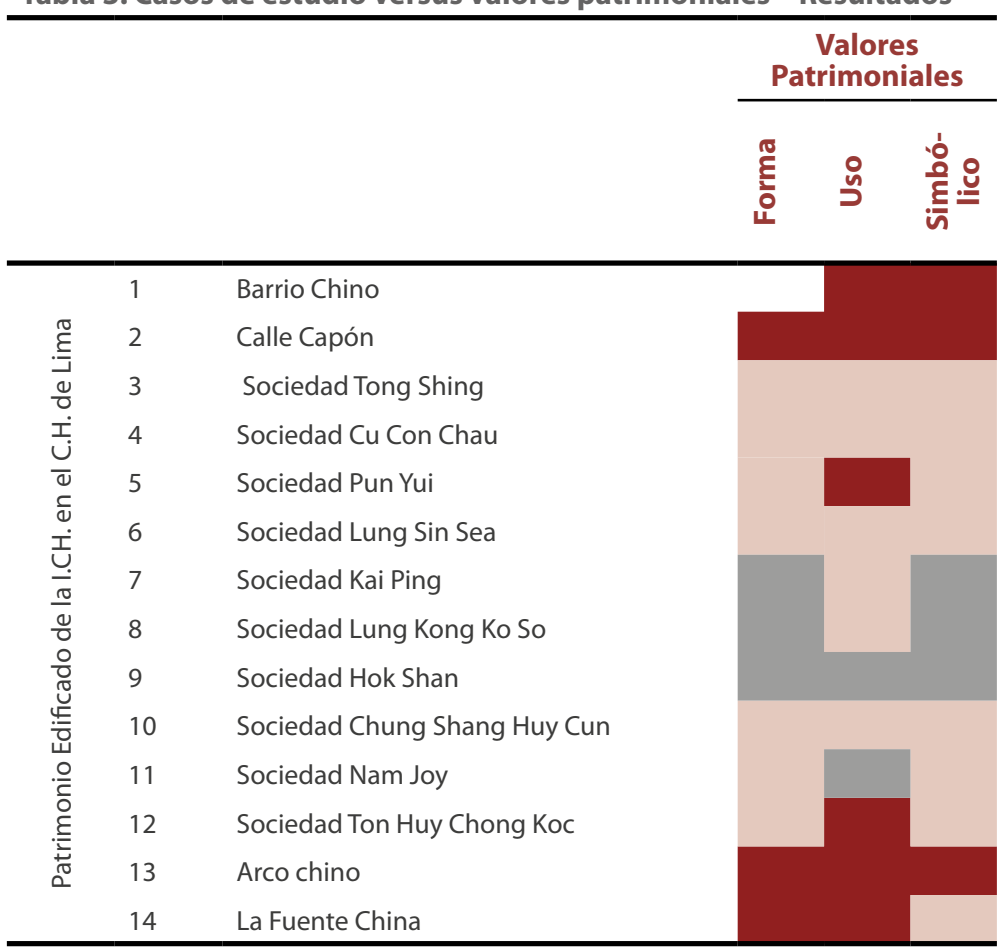

Leyenda

Tiene valor alto

Tiene valor medio

Tiene valor bajo

Elaboración propia, 2018.

Las Sociedades son edificios de corte occidental que presentan únicamente ideogramas -en relieves o en carteles- como única característica oriental, la mirada externa no los identifica como chinas; esto es potenciado por las restricciones de acceso. Sin embargo, los sujetos patrimoniales que han tenido acceso a espacios, saben valorar su originalidad por el equipamiento y las reliquias que se hallan dentro de estos inmuebles.

Valor formal - de conjunto 
Los sujetos patrimoniales presentan opiniones divididas sobre el conjunto: para la mirada externa, las edificaciones son percibidas como no integradas a su entorno; sin embargo, los sujetos patrimoniales que tienen una relación directa con las Sociedades (mirada interna) sí las encuentran integradas al Barrio Chino.

Valor de uso - económico

Los sujetos patrimoniales no vinculados directamente a la comunidad china que proviene de la inmigración consideran que las Sociedades no generan un valor económico en sí mismas, salvo por aquellas que optaron por implementar tiendas debido a su emplazamiento en una zona comercial; sin embargo, los académicos y expertos en el tema ven en las Sociedades Chinas suficiente potencial para poder generar dinero sin la necesidad del giro comercial.

Valor de uso - funcional

Debido a las restricciones de acceso a las Sociedades Chinas, se toma solo la opinión de los sujetos patrimoniales que provienen de la inmigración, así como de expertos en el tema. La mayoría de las Sociedades Chinas mantienen su función original: espacios de reunión para los miembros, lugar de culto, vivienda, etc.; sin embargo, el cambio generacional, la ubicación comercial y las dificultades para auto sostenerse culminaron en modificaciones para abrir más espacios comerciales.

Valor de uso - educativo

Las opiniones respecto a lo educativo son positivas en su mayoría, pero están dividas en dos puntos de vista: una parte de los sujetos patrimoniales considera que tienen potencial debido a que asocian los bienes inmuebles a la inmigración china y la otra parte se enfoca en el turismo cultural gastronómico debido a que comidas como el dim sum y celebraciones tradicionales como el Año nuevo chino o el Festival de la Luna son costumbres que ya forman parte de su vida cotidiana.

Valor de uso - social

La mirada externa considera que las Sociedades Chinas son poco accesibles sin entender específicamente el porqué ya que se presenta una dicotomía en la vida cotidiana: por un lado, los chinos son accesibles en su trato debido al comercio; sin embargo, debido a su cultura, su vida familiar o personal -estrechamente ligadas a las Sociedades Chinas- es celosamente mantenida en privado.

Valor de uso - científico

Desde la mirada externa no se considera que los bienes inmuebles tengan valor científico debido a que lo relacionan directamente con las ciencias exactas como Química, Física o Matemática. Por otro lado, los académicos y expertos en el tema consideran como valor científico a las ciencias humanas y sociales.

Valor simbólico - histórico

El bien patrimonial es testigo único de determinado suceso, en este caso la inmigración china al Perú; sin embargo, la mirada externa no considera a las Sociedades como un hecho importante en la historia peruana, según la muestra se debe al desconocimiento general sobre la inmigración china al Perú por una falta de inclusión o mayor espacio en el currículo nacional en educación básica.

Valor simbólico - de existencia

En este tema concordaron todos los sujetos patrimoniales en reconocer la representatividad de las Sociedades Chinas. Según la muestra, en el caso de la mirada externa, si 
bien se reconocen como no relacionados a este patrimonio, consideran su importancia para algún otro grupo social, por lo que consideran importante su preservación.

Valor simbólico - de legado

Similar a lo sucedido con el valor simbólico - de existencia, la mirada externa considera los bienes patrimoniales como legado cultural para determinado grupo social.

Valor simbólico - de identidad

La población peruana no se siente identificada con las Sociedades Chinas, los únicos que tienen un sentido de identidad son los que pertenecen a la comunidad que deviene de la inmigración (mirada interna) pese a que no necesariamente están relacionados con un bien específico.

Valor simbólico - de origen

La mirada externa no reconoce patrones chinos en las fachadas debido a que estos inmuebles presentan limitados elementos característicos de lo oriental. Por el contrario, y como ya se había mencionado antes, solo los sujetos patrimoniales que provienen de la migración tienen acceso a las diferentes Sociedades Chinas donde sí se puede apreciar la relación del bien patrimonial y su origen.

- Sobre la zona monumental, el ambiente urbano monumental y los hitos urbanos

Valor formal

La mirada académica tanto interna como externa reconoce el valor de conjunto como el valor formal más estimado reconociendo que la casuística urbana se integra plenamente al paisaje. A su vez, la casuística con los mejores índices fue la fuente china reconociendo que cuenta con importancia del diseño, relevancia de su concepción y construcción y se destaca significativamente en relación a otras construcciones del mismo tipo en: estilo, constructor y periodo.

Valor de uso

La mirada académica tanto interna como externa da razón a los valores funcional, educativo y social como los valores de uso más trascendentales. Y reconocen al Arco Chino como la muestra que genera mayor valor económico, que cuenta además con pleno potencial para el turismo cultural y concientización sobre la cultura e historia y finalmente mantiene una plena interacción social con la comunidad y fomenta una identidad sociocultural.

Valor simbólico

La mirada académica tanto interna como externa subraya que el valor de legado es el valor simbólico-significativo más resaltante. Y reconoce a los hitos urbanos -Arco Chino y fuente china- como las muestras que contribuyen significativamente a referenciar un momento determinado de la historia, favorecen al goce por su simple existencia, cuentan valor para las futuras generaciones y fomentan el reconocimiento relacionado a los lazos emocionales de la sociedad.

\section{A modo de conclusión}

La investigación cuestiona si existe el patrimonio edificado de la inmigración china en el centro histórico de Lima, según la definición de Howard sobre las escalas de patrimonio - formal e informal- se puede afirmar que: 
devenir Vol. 7, Nº14, JULIO-DICIEMBRE 2020, PP. 1155-172 - EsTUDIOS ISSN 2312-7562 E-ISSN 2616-4949

UNIVERSIDAD NACIONAL DE INGENIERÍA, LIMA

doi: https://doi.org/10.21754/devenir.v7i14.836

Existe solo un caso de patrimonio edificado formal de la inmigración china en el centro histórico de Lima y corresponde en específico a la fuente china ubicada en el parque de la Exposición. Por el contrario, el Ministerio de Cultura no reconoce al Barrio Chino como Zona Monumental, tampoco a la Calle Capón como Ambiente Urbano Monumental, ni le otorga a las Sociedades Chinas el valor de Monumento. La Municipalidad de Lima le reconoce la mitad de las Sociedades Chinas un Valor Monumental -Sociedades: Nam Joy, Tong Shing, Pun Yui, Chung Shan Huy Cun y Hok Shan- y la otra mitad se encuentra identificada como bien de entorno-Sociedades: Cu Con Chau, Lung Sin Sea, Kai Ping, Lung Kong Ko So y la Sociedad Central de Beneficencia China- y, finalmente, el Arco Chino no es un bien declarado patrimonio por el Ministerio de Cultura.

Si bien el patrimonio edificado de la inmigración china no representa todavía un patrimonio formal, representa un patrimonio informal de vital importancia para las comunidades que provienen de la inmigración china, así como para la población peruana que tiene presente al Barrio Chino como parte importante en la imagen de la ciudad, pero desde una escala urbana donde los hitos forman parte vital de este imaginario.

Se evidencia que, a raíz de la ubicación comercial de los diferentes edificios patrimoniales, la velocidad con la que se dan las transformaciones sin intervención técnica atenta contra su supervivencia, con ello se confirma que todas las Sociedades Chinas necesitan herramientas de gestión para evitar las modificaciones sin consulta especializada con el fin de no desaprovechar los valores que se mantienen. A la fecha las modificaciones y el descuido de dicho patrimonio ha devenido en la pérdida del bien patrimonial como por ejemplo los casos de la Sociedad Nam Joy que en la actualidad solo conservaba la fachada y de la Sociedad Hok Shan que cuando se desalojen los artículos del segundo piso pasará a ser un solo recuerdo para aquellos que relacionaban esos espacios con la inmigración china al Perú.

\section{Referencias}

Ballart H., \& Tresserras J. (2001). Gestión del patrimonio cultural. Barcelona: Editorial Ariel Patrimonio.

Chuhue R., Jing L., Coello A. (2012). La inmigración China al Perú. Arqueología, Historia y Sociedad. Lima: Fondo Editorial del Congreso del Perú.

Chuhue R., \& Li J. (2016). El Museo de la Sociedad Pun Yui del Perú. Illapa Mana Tukukuq, (10), 110117.

Chuhue R. (2016). Capón. El barrio chino de Lima. Lima: Municipalidad Metropolitana de Lima.

Feliu, J. (2014). Propuestas para una epistemología del patrimonio. Devenir, 1(2), 10-26.

Hayakawa, J. (2012). Restauro-UNI. Lima: Editorial Universidad Nacional de Ingeniería.

Lausent-Herrera, I. (2000). Sociedades y Templos chinos en el Perú. Lima: Fondo Editorial del Congreso del Perú.

Lynch, K. (1984). La imagen de la ciudad. Barcelona, España: Editorial Gustavo Gili.

Rodríguez, H. (2001). Hijos del celeste imperio en el Perú (1850-1900) Lima: Sur casa de estudios del socialismo. Segunda Edición.

Villamón, T. (2017). Reflexiones teóricas contemporáneas sobre patrimonio edificado y su significado. Devenir, 4(8), 123-133. 